\title{
Relationships between obsessive-compulsive disorder, depression and functioning before and after exposure and response prevention therapy
}

\author{
Sarosh J. Motivala, Ph.D. ${ }^{1,{ }^{*}}$, Maria Arellano, M.S. ${ }^{1}$, Rebecca Greco, M.S. ${ }^{1}$, David Aitken, \\ Psy.D. ${ }^{1}$, Nathan Hutchenson, Ph.D. ${ }^{1}$, Reza Tadayonnejad, M.D., Ph.D. ${ }^{1}$, Joseph O'Neill, \\ Ph.D. ${ }^{1}$, and Jamie D. Feusner, M.D. ${ }^{1}$ \\ ${ }^{1}$ UCLA Semel Institute for Neuroscience and Human Behavior
}

\begin{abstract}
Objective-Obsessive-compulsive disorder (OCD) is associated with impaired functioning and depression. Our aim was to examine relationships between OCD symptoms, depression, and functioning before and after exposure and response prevention (ERP), a type of cognitivebehavioral therapy for OCD, specifically examining whether functioning, depression and other cognitive factors like rumination and worry acted as mediators.
\end{abstract}

Methods-Forty-four individuals with OCD were randomized to four weeks of intensive ERP treatment first $(n=23)$ or waitlist then treatment $(n=21)$. We used a bootstrapping method to examine mediation models.

\begin{abstract}
Results-OCD symptoms, depression and functioning significantly improved from pre- to postintervention. Functioning mediated the relationship between OCD symptoms and depression and the relationship between functioning and depression was stronger at post-treatment. Depression mediated the relationship between OCD symptoms and functioning, but only at post-intervention. Similarly, rumination mediated the relationship between OCD symptoms and depression at postintervention.
\end{abstract}

Conclusions-Our findings suggest that after ERP, relationships between depression and functioning become stronger. Following ERP, treatment that focuses on depression and functioning, including medication management for depression, cognitive approaches targeting rumination, and behavioral activation to boost functionality may be important clinical interventions for OCD patients.

\section{Objective}

Depression is a significant problem in obsessive-compulsive disorder (OCD). Of all the mental health comorbidities associated with OCD, depression is the most common; more

\footnotetext{
*Corresponding author: Sarosh J. Motivala, Ph.D., 300 Medical Plaza, Room 2133, Los Angeles, California 90095-7057, Tel: 310 794-7315, Fax: 310 206-2802, smotivala@mednet.ucla.edu.

Disclosure of Interest

We wish to confirm that there are no known conflicts of interest associated with this publication and there has been no significant financial support for this work that could have influenced its outcome.
} 
than $50 \%$ of OCD patients meet lifetime criteria for major depressive disorder or dysthymia (Ruscio et al. 2010). In approximately $65 \%$ of these cases OCD preceded the onset of the depressive disorder or they developed in same year. Moreover, depression can significantly worsen OCD treatment outcomes (Overbeek et al. 2002). What accounts for this substantial comorbidity is not clear: is it the severity of the obsessions and compulsions themselves, the profound disruption in functioning, or some combination? With its laborious and often embarrassing compulsions paired with intense emotional disruption, OCD exerts profound effects on one's family, friendships, and school and work success and even daily living activities. Even after successful treatment for OCD, social impairments often remain: the number of individuals with OCD that are celibate, living alone and/or have impaired global assessment of functioning scores is considerably higher when compared to the normative population (Thomsen 1995). In cross-sectional studies with OCD patients, poor global functioning has been significantly associated with increased depressive symptoms (Abramowitz et al. 2007). Does OCD exert an effect on depression and is this effect mediated by functioning? Are these relationships stable or can they vary, especially when individuals get treatment?

Naturalistic cross-sectional studies provide important static images of these relationships, whereas prospective studies provide a "time-lapse" perspective, but intervention studies offer a unique possibility to uncover dynamic relationships of these variables. For example, when treatment results in lessening of OCD symptoms, this affords an opportunity to look at the resulting impact on functioning and depression.

Exposure and response prevention (ERP) is a type of CBT for OCD. Compared with medication treatments, ERP achieves more rapid therapeutic responses, which persist after discontinuation of therapy (Abramowitz 1998). Multiple studies suggest that ERP yields larger therapeutic effects than serotonin-reuptake inhibitor medication treatment (Romanelli et al. 2014; Skapinakis et al. 2016). Intensive ERP treatment improves not only OCD symptoms but reduces depressive symptoms too. Improvements in OCD symptoms seem to predict reductions in depressive symptoms during ERP and pharmacotherapy (Meyer et al. 2014); however, reductions in depressive symptoms do not appear to mediate reductions in OCD symptoms (Zandberg et al. 2015). Improvements in depressive symptoms could be secondary to improvements in OCD symptoms, and/or improvements in global functioning that are themselves the result of lessening of OCD symptoms.

As an exploratory set of mediation analyses, we examined rumination and worry and their role in the relationship between OCD symptoms and depression. Rumination plays an important contributory role in depression (Nolen-Hoeksema et al. 2008) and is associated with OCD diagnosis even after controlling for depressive symptoms (Abramowitz et al. 2007). Similarly worry is a common symptom across anxiety disorders (Olatunji et al. 2010) and in both those with OCD and those with depression (Brown et al. 1992). As noted by Clark (Clark 2004), OCD patients often have meta-cognitive beliefs that distressing thoughts are meaningful, have detrimental consequences to one's sense of self and that intrusive thoughts can and should be controlled. These beliefs in turn trigger repetitive thinking like worrying and ruminating as a potential strategy to manage the distressing thoughts. The frequent use of these repetitive thinking strategies may confer a cognitive vulnerability that 
might explain how OCD can lead to depression symptoms. We sought to explore whether worry and rumination would significantly mediate the connection between OCD symptoms and depression pre- vs. post-intervention.

The purpose of the current study was to examine pathways between OCD, depression and functioning and to examine whether the nature of these pathways change following treatment for OCD. Specifically, we wanted to test the hypothesis that global functioning mediates the association between OCD symptoms and depression. We predicted that this mediation relationship would be present both prior to and following treatment. Secondarily, we rotated this model to test whether depression mediated the relationship between OCD and functioning. Lastly, as exploratory analyses, we tested whether rumination and/or worry mediated relationships between OCD and depression before and after treatment.

\section{Methods}

\section{Participants}

Forty-four men and women with OCD between the ages of 18-60 participated in this study after providing informed consent. The UCLA Institutional Review Board approved the study. Participants were recruited from UCLA clinics, local psychiatrists, psychotherapists, flyers, and internet advertisements. To determine diagnostic eligibility, one of the study authors and licensed clinician (JDF) conducted detailed structured interviews with each potential participant. Primary OCD and comorbid diagnoses were determined using the ADIS-IV-Mini (Di Nardo et al. 1994). Participants were eligible if they scored $\geq 16$ on the Yale-Brown Obsessive Compulsive Scale (YBOCS) (Goodman et al. 1989). Comorbid anxiety disorders were allowed as long as OCD was the primary diagnosis. Comorbid major depressive disorder (MDD), dysthymic disorder, or depressive disorder not otherwise specified was allowed, but individuals were excluded if the ADIS-IV clinical significance rating was 6 or higher (severe).

Exclusion criteria included: 1) a lifetime diagnosis of psychotic disorder, bipolar disorder, lifetime substance dependence, or pervasive developmental disorder; 2) current diagnoses of attention deficit hyperactivity disorder, primary diagnosis of hoarding disorder, or any current comorbid psychiatric or medical condition that would have warranted immediate initiation of treatment that could interfere with study treatment; 3) severe OCD that warranted immediate inpatient psychiatric intervention; 4) currently consuming more than 7 units of alcohol per week; 5) prior treatment of more than 30 hours of CBT as part of an outpatient, inpatient, residential or intensive program that included exposure, response prevention, in session exposure, and development of a hierarchy; 6) IQ $<80$ on Wechsler abbreviated scales of intelligence; 7) being currently pregnant; 8) medications for medical conditions that have psychoactive properties (e.g. thyroid hormone); 9) and because all individuals also participated in a neuroimaging study, presence of metal or electronic implants in the body.

Regarding medication use, individuals were excluded if they were taking any class of medication other than serotonin-reuptake inhibitors (SRIs, including clomipramine); if on an SRI, individuals had to be on a stable dose for at least 12 weeks. Use of anxiety medications 
were allowed only if taken as needed but not on day of scan or during the course of treatment when exposures were conducted.

\section{Procedures}

This study was a randomized waitlist control trial of CBT, specifically ERP combined with neuroimaging assessments pre- and post-treatment, which have previously been described (Feusner et al. 2015; O'Neill et al. 2016). OCD patients were randomized to either a treatment first or waitlist first condition. In the latter condition, participants waited for four weeks and then received ERP. Treatment period was for four weeks for both groups. There were three assessment points in the study. At time 1, all participants received the battery of measures. After 4 weeks, at time 2, treatment-first participants received their post treatment assessment and waitlist first participants received their post waitlist assessment. The waitlist first group then received treatment and post treatment assessment at time 3 . The study also included standardized assessment in a non-OCD, healthy control group assessed at time 1 and time 2, but these data are not reported in the current paper.

\section{Intervention}

Participants received 90-minute sessions of ERP treatment 5 days a week for 4 weeks. Two licensed therapists provided treatment using a manualized approach (Kozak and Foa 1997a; Kozak and Foa 1997b). Both had received 3 or more years of specialty training in the UCLA OCD Intensive Treatment Program under the supervision of JDF and other senior therapists in addition to 3 or more years of training in outpatient ERP for OCD.

\section{Treatment quality assurance}

Therapy sessions of participants who provided consent (61\%) were videotaped and an independent evaluator (also a trained ERP therapist with 8 years experience treating OCD) rated all sessions for quality assurance, including adherence to the treatment manual and overall quality of the session. Average treatment adherence was rated at $97.7 \%$ and average quality of sessions was rated at 9.96 (0 to 10$)$.

\section{Assessments}

Interview Measures-An independent evaluator not involved in treatment or initial assessment carried out all interviews. The YBOCS (Goodman et al. 1989) is a semistructured clinical interview for assessing symptom severity in OCD. The measure has ten items, scored 0 to 4 resulting in a range of scores from 0-40 points. Its items assess distress, frequency, interference and resistance to obsessions and compulsions. The YBOCS is a widely used severity measure in clinical trials with OCD patients (Benito and Storch 2011). A number of clinical trials have set a minimum of 16 for inclusion criteria, and scores between 20-29 reflecting moderate symptoms, 30-40 reflecting severe symptoms (Ruscio et al. 2010). For depressive symptom severity, we used the Montgomery-Asberg Depression Rating Scale (MADRS), a ten-item interview with scores that range from 0-60 (Montgomery and Asberg 1979). The MADRS is an oft used assessment in clinical trials with good sensitivity to changes in symptoms (McDowell 2006). Scores below 6 suggest absence of depression, 7-19 reflect mild depression and scores from 20-34 representing 
moderate depression; scores above 35 on this measure are suggestive of severe depression (Snaith and Taylor 1985). To measure anxiety we used the Hamilton Anxiety Scale (HAMA) (Hamilton 1959). The HAMA is used to measure anxiety severity across different anxiety disorders. It has 14 items and scores range from 0-56. Scores are summed to form a total with scores between 0 and 5 representing little/no anxiety; 6 to 14 suggests mild anxiety, and scores of 15 or higher indicate severe anxiety (Hamilton 1959). We used the Global Assessment Scale (GAS) as our measure of general functioning and social and occupational performance (Endicott et al. 1976). The GAS ranges in scores from 0-100, with scores above 81 reflecting good or superior functioning in social, occupational, or school settings, 71-80 representing slight impairments, 61-70 representing some difficulties in functioning, 51-60 representing moderate impairments, $41-50$ serious impairments, $11-40$ a range of gross to major impairments.

Self-Report measures-We used the Penn State Worry Questionnaire (PSWQ) (Meyer et al. 1990), and The Ruminative Responses Scale (RRS) (Treynor et al. 2003) as estimates of cognitive processes involved in both OCD and depression. The PSWQ is a 16-item questionnaire of worry, with scores ranging from 16-80. Higher scores indicate greater tendency to worry with scores above 60 indicating severe anxiety. Brown and colleagues found that mentally healthy controls had a mean score of 35 and OCD patients had a score of 60 (Brown et al. 1992). The RSS is a 22-item measure of rumination, defined as a process of repetitively attending to the symptoms, possible causes and consequences of one's distress (Treynor et al. 2003). Higher scores indicate more rumination; scores can range from 22-88 and in a normative sample, mean scores on the RSS were 42 for women and 40 for men (Nolen-Hoeksema et al. 1999).

\section{Statistical Analyses}

In our statistical approach we examined demographic and baseline psychometric differences between the treatment first and the waitlist first groups using one-way Analysis of Variance (ANOVA). Next, we conducted a $2 \times 2$ repeated measures ANOVA examining clinical outcomes, with group (treatment first, waitlist first) as the between-group factor and time (time 1, time 2) as the within-group factor. At time 1 both groups received a baseline assessment and at time 2 the treatment first group had just finished the ERP intervention and the waitlist first group had just completed its waiting period. Our outcome measures were YBOCS, MADRS, GAS, HAMA, PSWQ, and RRS.

We analyzed mediation at two time points: baseline and post treatment. We pooled data from both groups at time 1 (pre-intervention), and time 2 for the intervention first group and time 3 for the waitlist first group (post-intervention). Only the waitlist first group received a time 3 assessment, occurring after they underwent the treatment protocol after the initial waiting period between times 1 and 2. We used Hayes's method of ordinary least squares regression (OLSR)-based path analysis for mediation analyses via the SPSS PROCESS macro, which includes bootstrap-based confidence intervals calculation (Hayes 2013; Hayes and Rockwood 2016). In this approach, the direct effect of X (the predictor variable) on $Y$ (the outcome variable) and indirect effect of $\mathrm{X}$ on $\mathrm{Y}$ through $\mathrm{M}$ (mediator) were calculated. There was significant mediation if a) total effect of $\mathrm{X}$ on $\mathrm{Y}$ (direct effect of $\mathrm{X}$ on $\mathrm{Y}+$ 
indirect effect of $\mathrm{X}$ on $\mathrm{Y}$ through $\mathrm{M}$ ) was significant AND b) the contribution of indirect effect in total effect (indirect effect of $\mathrm{X}$ on $\mathrm{Y}$ through $\mathrm{M}$ ) was significant. In PROCESS, the indirect effect is significant if the range between the lower and upper limits of the confidence interval did not include zero. For all mediation analyses, bias-corrected confidence intervals were determined using bootstrapping method implemented in PROCESS macro based on 10,000 bootstrap samples.

The specific mediation analyses tested whether: 1) GAS mediated the relationship between YBOCS and MADRS, at pre-intervention and post-intervention (our hypothesis); 2) MADRS mediates the relationship between YBOCS and GAS (termed the "rotated model"). Lastly, as exploratory analyses, we examined whether RSS and/or PSWQ mediate the relationship between YBOCS and MADRS.

\section{Results}

\section{Demographics \& Intervention effects}

Table 1 presents basic demographic and psychometric information. Table 2 represents the percentages of participants with specific comorbid diagnoses. Of the total sample, 15 had one comorbid disorder, 8 had two comorbid disorders, and 6 had three or more comorbid disorders. Also of the total sample, 11 had a comorbid depressive disorder and 22 had one or more comorbid anxiety disorders. There were significant time effects for YBOCS, MADRS, GAS, HAMA, PSWQ and RSS scores, as indicated in Table 3. From pre- to postintervention, scores significantly improved for each of these variables.

\section{GAS as mediator between YBOCS and MADRS scores}

At pre-intervention, our mediation analysis was significant; total effect value $=.83, \mathrm{p}=$. 0053. GAS significantly mediated the effect of YBOCS on MADRS; indirect effect value $=$ 0.46 . The range of the lower and upper confidence intervals did not include zero, indicating that the indirect effect was significant; the lower level for confidence interval (LLCI) was 0.01 and the upper level for confidence interval (ULCI) was 0.89 .

Similarly, at post-intervention, GAS was a significant mediator. The total effect was significant (effect value $=1.08, \mathrm{p}<.0001$ ) and the indirect effect was significant as well $($ effect value $=.18$, LLCI $=0.01$ and $\mathrm{ULCI}=0.47)$

\section{MADRS as a mediator between YBOCS and GAS}

Next, we rotated our model such that GAS became our outcome variable and MADRS became our mediator. At pre-intervention, MADRS was not a significant mediator. The total effect was significant (effect value $=-1.07, \mathrm{p}<.0001$ ) but the indirect effect was not (effect value $=-0.21, \mathrm{LLCI}=-0.68$ and $\mathrm{ULCI}=0.00$ ).

However, at post-intervention MADRS was a significant mediator. The total effect was significant (effect value $=-1.05, \mathrm{p}=.003$ ) and the indirect effect was significant (effect value $=-0.49 ;$ LLCI $=-0.96$, ULCI $=-0.1)$. 


\section{Exploratory mediational analyses: cognitive processes of rumination and worry}

We next wanted to examine rumination (measured with the RRS) and worry (measured with the PSWQ) as potential mediators between YBOCS and MADRS. In this sample, data collection for these measures started after the project was already underway, and as such, we had data for a smaller sample: RRS $n=34$; PWSQ $n=29$.

For RSS, at pre-intervention there was no significant mediation effect. The total effect of YBOCS and RSS on MADRS was not significant (effect value $=.21, \mathrm{p}=.42$ ). At postintervention however, RSS was a significant mediator. The total effect was significant (effect value $=1.14, \mathrm{p}=.0001)$ as was the indirect effect (effect value $=.21, \mathrm{LLCI}=.0019$, ULCI $=.693$ ).

For PSWQ, at pre-intervention there was no significant mediation. The total effect was significant (effect value $=.69, \mathrm{p}=.04$ ) but the indirect effect was not (effect value $=-.007$, $\mathrm{LLCI}=-.318, \mathrm{ULCI}=.065)$. At post-intervention there was no significant mediation. Similar with pre-intervention, the total effect was significant (effect value $=1.16, \mathrm{p}<.0001$ ) but the indirect was not (effect value $=-.003, \mathrm{LLCI}=-.120, \mathrm{ULCI}=.076$ ).

\section{Post hoc analyses of pairwise relationship changes from pre- to post-treatment}

Due to the observation that the mediation models involving YBOCS, MADRS, and GAS changed from pre- to post-treatment, we explored whether the pairwise relationships between these variables significantly changed across these time points. We used linear mixed models with MADRS as the dependent variable, time point as the repeated measure; subject as the random factor; and time point (pre- and post-treatment), GAS, and the interaction between time point and GAS as the fixed effects. There was a significant interaction between time point and GAS $\left(\mathrm{F}_{154.35}=4.72, \mathrm{p}=.034\right)$. The strength of the relationship between GAS and MADRS was stronger post-treatment than pre-treatment $(\mathrm{t}=$ $2.17, \mathrm{p}=.034)$. We repeated the analysis with YBOCS instead of GAS, but found no significant interaction between time point and YBOCS $\left(\mathrm{F}_{1} 54.00=1.08, \mathrm{p}=.30\right)$; neither was there a significant interaction effect when GAS was the dependent variable and YBOCS the predictor $\left(\mathrm{F}_{151.83}=1.71, \mathrm{p}=.20\right)$.

We conducted similar analyses to examine whether the RRS showed different strengths of relationships depending on time point, but found no significant interaction effect for MADRS $\left(\mathrm{F}_{149.55}=.46, \mathrm{p}=.50\right)$ or for YBOCS $\left(\mathrm{F}_{141.41}=.15, \mathrm{p}=.70\right)$. Thus, only the relationship between GAS and MADRS significantly changed, becoming significantly stronger post-treatment compared to pre-treatment.

\section{Medication status}

Fourteen of the 44 participants were taking serotonin reuptake inhibitor medications, which can influence both OCD and depression symptoms (the medication status and doses were required to be the same pre-treatment and post-treatment). We therefore repeated all analyses using medication status as a covariate, but found no significant effects of medication status (all p's $>.05$ ) and no significant changes in the overall mediation or regression models. 


\section{Conclusion}

The effectiveness of ERP in treating OCD in our study was consistent with the literature. CBT led to a $41 \%$ reduction in YBOCS scores and significant reductions in depressive (MADRS) and general anxiety symptoms (HAMA), worrying (PSWQ), and global functioning (GAS). MADRS scores fell as well but scores were in the mild range to begin with, likely because we deliberately excluded individuals with ADIS-IV depressive symptom clinical significance ratings of 6 or higher (indicative of severe depressive symptoms). Functioning scores shifted from the moderate to mild range at post-treatment.

In our study, functioning mediated the relationship between OCD symptoms and depression both before and after treatment. This suggests that OCD symptoms can both directly impact depression and indirectly do so by impairing functioning. The mediating effect of functioning held up even after four weeks of five days per week ERP. Thus, functioning clearly seems to play a role in affecting depression. Clinicians could use this indirect pathway as an additional treatment target -specifically addressing deficits in functioning. Behavioral activation is a type of behavior therapy typically carried out in patients with unipolar depression; it is a treatment approach that focuses on increased functional activity as a strategy to reduce depressive symptoms (Dimidjian et al. 2006).

When we rotated our model so that functioning was the outcome and depression was the mediator, we found that depressive symptoms mediated the relationship between OCD symptoms and functioning at post-intervention, but not initially at pre-intervention. These results suggest that even mild, post-ERP residual depressive symptoms impact level of functioning in OCD. Depressive symptoms commonly arise after the onset of OCD symptoms (Rasmussen and Tsuang 1986; Ruscio et al. 2010). Prevalence of depressive symptoms ranges from 21-50\% of OCD patients (Abramowitz 2004) and depressive symptoms typically correlate strongly with OCD in the range of Pearson correlations of $r=$. 60 (Goodman et al. 1989). It seems reasonable for clinicians to focus on applying ERP to reduce OCD symptoms with the expectation that depressive symptoms will follow suit. This appeared to be the case in our sample; OCD and depressive symptoms and functioning all improved. However, the nature of the relationships between functioning and OCD and depressive symptoms changed following treatment; the strength of the relationship between functioning and depression increased at post-treatment when OCD symptoms were reduced. This raises the question of targeting both depression and functioning following ERP-related improvements in OCD symptoms. Specifically, this could include behavioral activation to boost functionality and depressive symptoms, cognitive approaches targeting depressive thought patterns, and emphasizing medication management for depressive symptoms.

Cognitive approaches integrated with ERP have evidence of success in improving distress tolerance, reducing OCD symptom-related dysfunctional beliefs, and reducing dropout rates (Mckay et al. 2015). Behavioral activation is a viable treatment in depressive disorders (Cuijpers et al. 2007) but has not been examined in the context of anxiety disorders. Medication management for depression in OCD appears to be an important treatment option, but few studies have addressed the effects on depressive symptom improvement in OCD with medication management. Those that have, have had small sample sizes (Abramowitz 
2004). It is important to note that although about $32 \%$ of our sample was taking serotonin reuptake inhibitors, which are first-line treatments for both OCD and for depression, medication status did not affect the results of the mediation analyses. Thus, although some of the individuals on medications could have received partial benefit for OCD and/or depressive symptoms prior to the study, this did not appear to affect the inherent relationships between OCD, depression, and functioning. Those who had obtained more substantial benefits from medications could have been screened out for not meeting the inclusion criteria of YBOCS $\geq 16$; it is therefore still possible that medications could change these relationships in some individuals.

ERP led to significant reductions in two measures of repetitive, problematic thinking styles worry and rumination. For worry, baseline levels of the PSWQ were similar to other studies with OCD patients (Brown et al. 1992). Although scores fell by approximately 14\%, from 65 to 56, post intervention scores were still elevated in contrast to published scores for normative data (Brown et al. 1992). In contrast, rumination, measured with the RSS, was modestly elevated at baseline and fell to levels similar to those found in a normative sample (Nolen-Hoeksema et al. 1999).

Exploratory mediation analyses indicated that rumination was a mediator between OCD symptoms and depression but only at post-intervention. Worry was not a significant mediator of depressive symptoms either before or after the intervention. Our findings on rumination suggest that before treatment, when symptom severity is higher across the board, functioning is an important mediating variable between OCD symptoms and depression; however when individuals are improved following intensive treatment, rumination emerges as a mediator linking OCD and depressive symptoms. ERP does not overtly target depressive ruminating. In our sample, the tendency to ruminate did improve to levels seen in normative samples. These exploratory analyses suggest that when OCD symptoms are lower, rumination influences the relationship between OCD and depressive symptoms. If patients continue with outpatient (less frequent) therapy after completing intensive ERP, their tendency to ruminate may be an important intervention target to help further reduce depression. These conclusions should be framed with awareness that our sample was small and that only a subsample of participants received the rumination and worry questionnaires.

There are important caveats and limitations to this study. Our aim in this study was to identify if functioning, and other cognitive variables, play important contributory roles to the relationship between OCD symptoms and depression. Our preliminary findings suggest that they do. However, there are a number of caveats to our findings. Our study had a small sample size ( $\mathrm{N}=44)$ and our analyses were post-hoc and were not built into the design of the study. Although our study had two time points, our mediation analyses were cross-sectional and conclusions regarding causal pathways should include awareness of this limitation. Future studies using additional time points, perhaps at mid-intervention, will be useful in corroborating our findings. Our intervention did not explicitly test treatment that was constructed to address functioning or rumination. In the future, comparing treatment arms that include medication management specific for depression, or behavioral activation or CBT targeting rumination in combination with ERP would be informative. Future studies 
that do so would provide more direct evidence for routing the interplay between these variables.

A limitation that potentially affects the generalizability of the results is that we excluded individuals if their comorbid depressive disorder was rated severe or higher, or it was the primary diagnosis rather than $\mathrm{OCD}$, in order to minimize the impact of severe depression on brain function and structure given that all participated in a neuroimaging study. This may have affected the composition of our sample with respect to comorbid depression, as eight individuals (18.2\%) met criteria for MDD, while eleven (25\%) met criteria for any depressive disorder. This is slightly lower than other studies of current depressive disorder comorbidity in OCD, which have found rates of 22.4 to 32\% for MDD (Overbeek et al. 2002) and 37\% for any depressive disorder (Perugi et al. 1997). Thus, it is unknown if the current relationships found in this study among depression, OCD symptoms, and functioning would generalize to samples with a higher percentage of comorbid MDD, and/or more severe depressive symptoms. In addition, our study did not assess whether depressive symptoms developed prior to, or secondary to, their OCD symptoms. It is possible that depression that is secondary to OCD will remit more readily following ERP, but that primary depression may not. Few studies exist on this specific question and our findings strongly urge us to explore the interplay of OCD and depressive symptoms more thoroughly.

Also limiting generalizability to other treatment settings is the fact that we tested the effects of intensive ERP - 5 days per week for 4 weeks - whereas ERP in some clinical settings is delivered weekly or twice weekly, over several months. Whether the current findings would also be observed with less intensive ERP remains to be studied; however one might predict that once or twice weekly treatment that is spread out over a longer total number of weeks might allow more time for general functioning to gradually improve, as well as depression, and the relationships among these and OCD symptoms potentially could evolve and change. Another consideration regarding generalizability is that only $32 \%$ of the current sample was taking a medication for OCD, which is lower than that found in other clinical samples in the U.S. (e.g. 83.5\% (Patel et al. 2014) and 93\% (Blanco et al. 2006). Again, whether the relationships described in the current study would hold for OCD patients in general remains to be studied. Because the most commonly prescribed medications for OCD, SRIs, also reduce symptoms of depression, it is possible that the relationships between depression, OCD symptoms and functioning may be similar to our post-treatment findings.

In conclusion, our findings suggest that the relationship between OCD, functioning and depressive symptoms are not static. They clearly change over the course of treatment and as such, there may be important key time points that specific interventions that go beyond ERP and focus on functioning and depression may be beneficial for OCD patients.

\section{Acknowledgments}

The authors would like to thank Courtney Sheen for her diligence in handling administrative responsibilities associated with this project and and Michelle Massi and Natalie Abrahami for their excellent work as the treating therapists on this study. 


\section{References}

Abramowitz JS. Does cognitive-behavioural therapy cure obsessive-compulsive disorder? A metaanalytic evaluation of clinical significance. Behav Ther. 1998; 29:339-355.

Abramowitz JS. Treatment of obsessive-compulsive disorder in patients who have comorbid major depression. J Clin Psychol. 2004; 60:1133-1141. [PubMed: 15389622]

Abramowitz JS, Storch EA, Keeley M, Cordell E. Obsessive-compulsive disorder with comorbid major depression: What is the role of cognitive factors? Behav Res Ther. 2007; 45:2257-2267. [PubMed: 17521605]

Benito K, Storch Ea. Assessment of obsessive-compulsive disorder: review and future directions. Expert Rev Neurother. 2011; 11:287-98. [PubMed: 21306215]

Blanco C, Olfson M, Stein DJ, Simpson HB, Gameroff MJ, Narrow WH. Treatment of obsessivecompulsive disorder by U.S. psychiatrists. J Clin Psychiatry. 2006; 67:946-951. [PubMed: 16848654]

Brown TA, Antony MM, Barlow DH. Psychometric properties of the Penn state worry questionnaire in a clinical anxiety disorders sample. Behav Res Ther. 1992; 30:33-37. [PubMed: 1540110]

Clark, DA. Cognitive Behavioral Therapy for OCD. New York: The Guilford Press; 2004.

Cuijpers P, van Straten A, Warmerdam L. Behavioral activation treatments of depression: A metaanalysis. Clin Psychol Rev. 2007; 27:318-326. [accessed 2017 Jun 2] http://www.sciencedirect.com/ science/article/pii/S027273580600136X. [PubMed: 17184887]

Dimidjian S, Hollon SD, Dobson KS, Schmaling KB, Kohlenberg RJ, Addis ME, Gallop R, McGlinchey JB, Markley DK, Gollan JK, et al. Randomized Trial of Behavioral Activation, Cognitive Therapy, and Antidepressant Medication in the Acute Treatment of Adults With Major Depression. J Consult Clin Psychol. 2006; 74:658-670. [PubMed: 16881773]

Endicott J, Spitzer RL, Fleiss JL, Cohen J. The global assessment scale. A procedure for measuring overall severity of psychiatric disturbance. Arch Gen Psychiatry. 1976; 33:766-771. [PubMed: 938196]

Feusner JD, Moody T, Lai TM, Sheen C, Khalsa S, Brown J, Levitt J, Alger J, O’Neill J. Brain Connectivity and Prediction of Relapse after Cognitive-Behavioral Therapy in ObsessiveCompulsive Disorder. Front Psychiatry. 2015; 6:74. [accessed 2016 Jun 20] http:// www.frontiersin.org/Neuropsychiatric_Imaging_and_Stimulation/10.3389/fpsyt.2015.00074/ abstract. [PubMed: 26042054]

Goodman WK, Price LH, Rasmussen Sa, Mazure C, Fleischmann RL, Hill CL, Heninger GR, Charney DS. The Yale-Brown Obsessive Compulsive Scale. I. Development, use, and reliability. Arch Gen Psychiatry. 1989; 46:1006-11. [PubMed: 2684084]

Hamilton M. The assessment of anxiety states by rating. Br J Med Psychol. 1959; 32:s0-s5.

Hayes, AF. Introduction to meditaion, moderation, and conditional process analysis: A regressionbased approach. Guilford Press; 2013.

Hayes, AF., Rockwood, NJ. [accessed 2017 Mar 16] Regression-based statistical mediation and moderation analysis in clinical research: Observations, recommendations, and implementation. Behav Res Ther. 2016. http://linkinghub.elsevier.com/retrieve/pii/S0005796716301887

Kozak, MJ., Foa, EB. Mastery of obsessive-compulsive disorder: a cognitive-behavioral approach client workbook. New York: Oxford University Press; 1997a.

Kozak, MJ., Foa, EB. Mastery of obsessive-compulsive disorder: a cognitive-behavioral approach therapist guide. New York: Oxford University Press; 1997b.

McDowell, I. Measuring Health. 2006.

Mckay D, Sookman D, Neziroglu F, Wilhelm S, Stein DJ, Kyrios M, Matthews K, Veale D. Efficacy of cognitive-behavioral therapy for obsessive-compulsive disorder. Psychiatry Res. 2015; 225:236246. [PubMed: 25613661]

Meyer JM, McNamara JPH, Reid AM, Storch EA, Geffken GR, Mason DM, Murphy TK, Bussing R. Prospective Relationship Between Obsessive-Compulsive and Depressive Symptoms During Multimodal Treatment in Pediatric Obsessive-Compulsive Disorder. Child Psychiatry Hum Dev. 2014; 45:163-172. [accessed 2017 Jun 2] http://www.ncbi.nlm.nih.gov/pubmed/23756717. [PubMed: 23756717] 
Meyer TJ, Miller ML, Metzger RL, Borkovec TD. Development and validation of the penn state worry questionnaire. Behav Res Ther. 1990; 28:487-495. [PubMed: 2076086]

Montgomery SA, Asberg M. A new depression scale designed to be sensitive to change. Br J Psychiatry. 1979; 134:382-389. [PubMed: 444788]

Di Nardo, P., Brown, TA., Barlow, DH. Anxiety Disorders Interview Schedule for DSM-IV: Lifetime Version. Albany, NY: Graywind; 1994.

Nolen-Hoeksema S, Larson J, Grayson C. Explaining the gender difference in depressive symptoms. J Pers Soc Psychol. 1999; 77:1061-1072. [PubMed: 10573880]

Nolen-Hoeksema S, Wisco BE, Lyubomirsky S. Rethinking Rumination. Perspect Psychol Sci. 2008; 3:400-424. [PubMed: 26158958]

O’Neill J, Lai TM, Sheen C, Salgari GC, Ly R, Armstrong C, Chang S, Levitt JG, Salamon N, Alger JR, et al. Cingulate and thalamic metabolites in obsessive-compulsive disorder. Psychiatry Res Neuroimaging. 2016; 254:34-40. [PubMed: 27317876]

Olatunji BO, Wolitzky-Taylor KB, Sawchuk CN, Ciesielski BG. Worry and the anxiety disorders: A meta-analytic synthesis of specificity to GAD. Appl Prev Psychol. 2010; 14:1-24.

Overbeek T, Schruers K, Vermetten E, Griez E. Comorbidity of obsessive-compulsive disorder and depression: Prevalence, symptom severity, and treatment effect. J Clin Psychiatry. 2002; 63:11061112. [PubMed: 12523869]

Patel SR, Humensky JL, Olfson M, Simpson HB, Myers R, Dixon LB. Treatment of obsessivecompulsive disorder in a nationwide survey of office-based physician practice. Psychiatr Serv. 2014; 65:681-4. [PubMed: 24585056]

Perugi G, Akiskal HS, Pfanner C, Presta S, Gemignani A, Milanfranchi A, Lensi P, Ravagli S, Cassano GB. The clinical impact of bipolar and unipolar affective comorbidity on obsessive-compulsive disorder. J Affect Disord. 1997; 46:15-23. [PubMed: 9387083]

Rasmussen SA, Tsuang MT. Clinical characteristics and family history in DSM-III obsessivecompulsive disorder. Am J Psychiatry. 1986; 143:317-322. [PubMed: 3953865]

Romanelli RJ, Wu FM, Gamba R, Mojtabai R, Segal JB. Behavioral therapy and serotonin reuptake inhibitor pharmacotherapy in the treatment of obsessive-compulsive disorder: a systematic review and meta-analysis of head-to-head randomized controlled trials. Depress Anxiety. 2014; 31:64152. [accessed 2017 Jun 2] http://doi.wiley.com/10.1002/da.22232. [PubMed: 24390912]

Ruscio, A., Stein, D., Chiu, W., Kessler, R. [accessed 2016 Jun 16] The epidemiology of obsessivecompulsive disorder in the National Comorbidity Survey Replication. Mol Psychiatry. 2010. http:// www.nature.com/mp/journal/v15/n1/abs/mp200894a.html

Skapinakis P, Caldwell DM, Hollingworth W, Bryden P, Fineberg NA, Salkovskis P, Welton NJ, Baxter $\mathrm{H}$, Kessler D, Churchill R, et al. Pharmacological and psychotherapeutic interventions for management of obsessive-compulsive disorder in adults: a systematic review and network metaanalysis. The lancet Psychiatry. 2016; 3:730-9. [accessed 2017 Jun 2] http:// linkinghub.elsevier.com/retrieve/pii/S2215036616300694. [PubMed: 27318812]

Snaith RP, Taylor CM. Rating scales for depression and anxiety: a current perspective. Br J Clin Pharmacol. 1985; 19:17S-20S. [PubMed: 3994903]

Thomsen PH. Obsessive-compulsive disorder in children and adolescents. A 6-22 year follow-up study of social outcome. Eur Child Adolesc Psychiatry. 1995; 4:112-122. [PubMed: 7796249]

Treynor W, Gonzalez R, Nolen-Hoeksema S. Rumination reconsidered: A psychometric analysis. Cognit Ther Res. 2003; 27:247-259.

Zandberg LJ, Zang Y, McLean CP, Yeh R, Simpson HB, Foa EB. Change in obsessive-compulsive symptoms mediates subsequent change in depressive symptoms during exposure and response prevention. Behav Res Ther. 2015; 68:76-81. [accessed 2016 Jul 12] http://www.ncbi.nlm.nih.gov/ pubmed/25824533. [PubMed: 25824533] 


\section{Key Points}

The relationships between OCD symptoms, depression and functioning shift from pre- to post-treatment.

Functioning mediated the relationship between OCD and depressive symptoms both pre- and post-intervention. Post hoc analyses indicated the relationship between functioning and depression was stronger at post-intervention.

Depression was a mediator of OCD and functioning, but only at post intervention.

More fully integrating approaches that focus on depression and functioning, including medication management for depression, cognitive approaches targeting depressive thought patterns, and behavioral activation to boost functionality may be important clinical interventions for OCD patients, especially after ERP treatment. 


\section{Table 1}

\section{Demographics}

\begin{tabular}{ll} 
& $\mathbf{n}(\boldsymbol{\%})$ \\
\hline Age (mean years \pm std dev) & $33.6 \pm 11.2$ \\
Sex & \\
$\quad$ Men & $23(52 \%)$ \\
Women & $21(48 \%)$ \\
Ethnicity & \\
Caucasian & $30(68 \%)$ \\
Asian & $5(11 \%)$ \\
Black & $3(7 \%)$ \\
Hispanic & $6(14 \%)$ \\
Medication Use & \\
Medicated & $14(32 \%)$ \\
Unmedicated & $30(68 \%)$ \\
\hline
\end{tabular}

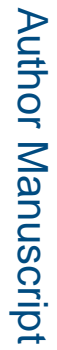

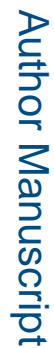

Int J Psychiatry Clin Pract. Author manuscript; available in PMC 2018 March 01. 


\section{Table 2}

\section{Comorbid Diagnoses for OCD participants}

\begin{tabular}{lc} 
Comorbid diagnosis & Number (Percentage) of Participants \\
\hline Social anxiety disorder & $17(38.6 \%)$ \\
Generalized anxiety disorder & $9(20.5 \%)$ \\
Major depressive disorder & $8(18.2 \%)$ \\
Specific phobia(s) & $6(13.6 \%)$ \\
Body dysmorphic disorder & $3(6.8 \%)$ \\
Dysthymic disorder & $2(4.5 \%)$ \\
Panic disorder & $2(4.5 \%)$ \\
Depressive disorder not otherwise specified & $1(2.3 \%)$ \\
Posttraumatic stress disorder & $1(2.3 \%)$ \\
\hline No comorbid diagnosis & $15(34.1 \%)$ \\
\hline
\end{tabular}

Note: total percentages for comorbid diagnoses do not sum to $100 \%$ because some participants had multiple comorbid diagnoses 


\section{Table 3}

Treatment Effects

\begin{tabular}{llcll} 
& Time 1 & Time 2 & F & p \\
\hline YBOCS & $24.9 \pm 4.5$ & $14.7 \pm 5.2$ & $\mathrm{~F}(1,43)=160$ & $<.0001$ \\
MADRS & $15.3 \pm 9$ & $10.6 \pm 8.9$ & $\mathrm{~F}(1,43)=12.8$ & $<.001$ \\
HAMA & $12 \pm 5.5$ & $8.2 \pm 5.1$ & $\mathrm{~F}(1,43)=19.4$ & $<.0001$ \\
GAS & $57.3 \pm 8.3$ & $69.4 \pm 13.2$ & $\mathrm{~F}(1,43)=75.2$ & $<.0001$ \\
PSWQ & $65.4 \pm 8.8$ & $56.4 \pm 9.8$ & $\mathrm{~F}(1,28)=25.5$ & $<.0001$ \\
RSS & $45.5 \pm 8.9$ & $38.6 \pm 11.3$ & $\mathrm{~F}(1,27)=12.14$ & $<.002$ \\
\hline
\end{tabular}

YBOCS=Yale-Brown Obsessive Compulsive Scale; MADRS=Montgomery-Asberg Depression Rating Scale; HAMA=Hamilton Anxiety Scale; GAS=Global Assessment Scale; PSWQ=Penn State Worry Questionnaire; RSS=Ruminative Responses Scale 